UC-15

Issued: July 1981

\title{
Analytical Techniques to Reduce the Number of Physical Siandards Needed for Neutron Coincidence Counters
}

\author{
Gerald E. Bosler
}

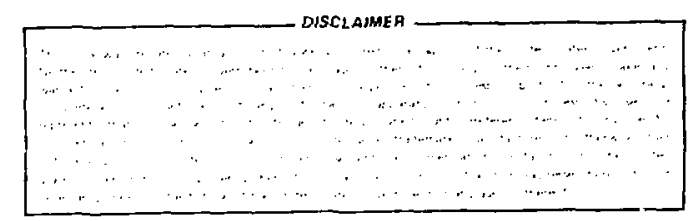




\title{
ANALYTICAL TECHNIQUES TO REDUCE THE NUMBER OF PHYSICAL STANDARDS \\ NEEDED FOR NEUTRON COINCIDENCE COUNTERS
}

\author{
by
}

Gerald E. Bosler

\begin{abstract}
A technique for performing calculationally assisted evaluations of shift-register coincidence counter data is discussed. An example of a typical application for some high mass $\mathrm{PuO}_{2}$ samples is presented. Methods for applying the technique to general coincidence counter use are described.
\end{abstract}

\section{INTRODUCTION}

The high-level neutr coincidence counter (HLNCC) ${ }^{\text {? }}$ and the active well coincidence cuunter ( $A W C C)^{2}$ have been developed at the Los Alamos National Laboratory, l.os Alainos) for use by International Atomic Ene 'g." Agency (IAEA) personnel in assaying materials containing plutonium and uranium. Both passive and active assaying capabilities are ava.'able with the HLNCC and AwCC. In the passive mode, plutonium materials with high spontaneous fission yield isotopes, that is, $238 \mathrm{Pu}, 240 \mathrm{Pu}$, and $242 \mathrm{pu}$, are assayed by determining coincident events between neutrons from spontaneous fission. Materials composed primarily of isotopes with low spontaneous fission yields such as ${ }^{235} U$ and ${ }^{233} U$ are assayed in the active mode by using small AmLi interrogation sources to induce fission in the sample. Active mode detector response results from coincident events between neutrons from induced fission. 
Ideally, a device such as the HLNCC or AWCC should exhibit linear response of count rate vs mass when used to assay unknown materials. For the HLNCC and AWCC over some limited material ranges, the response is linear. However, in other ranges, effects such as sample self-shielding and sample multiplication cause nonlinear responses. Such sample effects are further propagated in the count rate by the shift-register (SR) electronics circuitry ${ }^{3}$ used with the HLNCC and AWCC. As shown in Ref. 4, SR circuitry measures a response that is proportional to the neutron multiplicity in the sample. Samples with little or no multiplication will have smaller proportionality constants than samples with higher multiplication. As a result of these nonlinear effects, efficient and accurate operation of the HLNCC and AWCC depends on the availability of appropriate calibration techniques.

A combined calculation-experimental technique for assaying unknown samples in $S R$ coincidence counters is described in this report. The calculational portion of the technique uses a method developed by stewart ${ }^{5}$ to generate correction factor data that account for sample multiplication and SR effects on the coincidence count rates. Calculationally corrected count rates are relative quantities that must be normalized for the specific detector being used. The normalization is accomplished by obtaining a count rate in the detector of interest for a well-characterized material. from this count rate and the appropriate correction factor, the slope of a linear-corrected count rate vs mass curve is determined. With this value for the slope that is a function of the detector and type of material being measuied, unknown samples can be evaliated. The accuracy of the evaluation depends on how wel: the "known" normalizing material represents unknown samples. Thus, even though calculationally assisted calibration is possible for coincidence counters, the method still requires at least one experimental data point for normalization. 


\section{I. COINCIDENCE COUNTER CALIBRATION TECHNIQUES}

The simplest and most accurate calibration technique is to generate a calibration curve using various quantities of wellcharacterized materials. When such calibration curves adequately represent the composition and geometry of unknown samples, accurate assays (probably within 1 percent) are possible with the HLNCC and AWCC. IAEA inspectors encounter a wide variety of sample types. Typically, materials might be oxides, fluorides, or metals in powder, granular, or pellet form with varying amounts of impurities. Containers are not stanciard, varying in size and shape. Because most of these parameters affect sample multiplication and overall detector response, ideally a calibration curve representing each possible assay situation would be appropriate. Generating all such curves, however, is impractical because well-characterized standard samples for all materials are simply not available. Thus, alternative calibration techniques are needed.

One technique is to establish universal caibration curves that represent many sample types. For instance, a single calibration curve for $\mathrm{PuO}_{2}$ powder materials seems to be appropriate over a large range of masses regardless of the relative isotopic compositions. 6 Such a curve has been recently used to correctly assay several samples with compositions different irom the samples used in generating the curve. ${ }^{7}$ other types of $\mathrm{PuO}_{2}$ samples (that is, pellets), however, appear to have slightly different characteristics from powders, therefore requiring separate calibration curves. ${ }^{6}$

Another approach to calibrating coincidence counter data is to correct count-rate data for sample multiplication and shiftregister electronics effects using experimental techniques developed by Enssiin et al. ${ }^{5}$ With such correction factor techniques, coincidence count rates are adjusted to yield linear responses of corrected count rate vs sample mass. Application of experimentally determined correction factors have three potential limitations: (a) the technique is applicable only for passive assays of materials with well-defined compositions, (b) careful background measurements are necessary, and ( $c$ ) errors in the experimentally 
determined values begin to get very large when neutron multiplica$t$ ion exceeds 1.5 .8

Because experimentally determined parameters are used in either generating a calibration curve or using Enssion's method for correcting data, these two techniques should be used if at ali possible when evaluating unknown samples. In the event these two techniques are unavailable for a given sample material, ari alternate approach of using calculationally assisted calibration methods is proposed.

\section{I. CALCULATIONAL TECHNIQUES}

Two previously developed passive-assay calculational techniques 5,9 were used in this investigation. Bo:h methods, which use modified versions of the neutron-photon Monte Carlo code MCNP10 to predict different aspects of coincidence detector responses, were modified to include active-assay systems.

In the first technique, developed by MacDonald, 9 the SR electronic circuitry ${ }^{3}$ used for determining coincidence response is modeled calculationally. In this Monte Carlo simulation of SR circuitry, neutrons released from either spontaneous or induced fission are time-dependently transported to the ${ }^{3} \mathrm{He}$ detector tubes where ${ }^{3}$ He $(n, p)$ reactions are tallied. Time from birth in the sample to interaction with ${ }^{3}$ He is recorded for each neutron and then compared with similar times for other fission neutrons to determine coincident events. The model uses SR gate width, predelay, and deadtimes to predict as accurately as possible the coincidence response. For active assaying, this technique was modified to include effects of using AmLi neutrons to induce fissions in samples with low $(\alpha, n)$ and spontaneous fission yields. Aithough this calculational technique provides accurate results, 9 excessive computer execution times limit the usefulness. Generation of many calibration curves to satisfy the needs of IAEA inspectors is impractical because the total computer time required (even if readily available) would be prohibitively expensive. 
The second MCNP technique developed by Stewart $^{5}$ assumes a known detector response function 4 to calculate sample-multiplication correction factors. These correction factors are the ratio of the detector response with sample self-shielding and multiplication to the detector response without these effects. These correction factors are sample dependent and are oniy loosely (through backscattering from detector walls) coupled to detector geometries.

The techrique has been extended to include active assaying by redefining the denominator of the correction-factor term. For passive assays doscribed above, the denominator is defined as the coincidence response if all neutrons created in spontaneous $f$ issioning ieak out of the sample without further interactions; whereas, in the active mode, the denominator is defined as the coincidence response resulting from all neutrons created in fissions induced only by AmLi-source neutrons. The correction-factor technique requires much less computer time than the full coincidence response technique. For this reason the correction-factor approach is far more practical as a coincidence counter calibration tool.

One possible drawback to the correction-factor technique occurs in high-multiplying samples. In Ref. 4, the coincidence response for $n$ net neutrons leaking from the sample into the detector is shown to be proportional to

$$
n(n-1) p_{n} \text {, }
$$

where $P_{n}$ is the probability of $n$ neutrons leaking. Correction factors are calculated by MCNP by determining $P_{n}$ values for this coincidence response function. For low-multiplying samples, $n$ rarely exceeds 20 . The $P_{n}$ values for these samples are correctly predicted and the overall coincidence response function is well-characterized. In samples with high multiplications, $n>20$ leakage multiplication events are quite possible and potentially very important because of the $n(n-1)$ term in the coincidence response function. In MCNP, initial source event sampling is 
somewhat limited, resulting in poor sampling for leakage events with $n>20$. The calculated $p_{n}$ values for these highmultiplication samples are too large, resulting in significantly overpredicted correction factors. Techniques for correctly calculating $P_{n}$ for $n>20$ have been investigated with mixed success.

IV. VERIFICATION OF CORRECTION FACTOR TECHNIQUES FOR LARGE PUO 2 SAMPLES

The validity of Monte Carlo calculated correction factors for $\mathrm{PuO}_{2}$ samples with effective $240 \mathrm{pu}$ masses less than $250 \mathrm{~g}$ has been previously demonstrated by Stewart et al. ${ }^{5}$ However, because IAEA inspectors typically measure much larger $\mathrm{PuO}_{2}$ samples, verification of correction factor techniques for effective ${ }^{240} \mathrm{Pu}$ masses larger than $250 \mathrm{~g}$ is needed. Data for some typical $\mathrm{PuO}_{2}$ samples with oxide masses up to $3 \mathrm{~kg}$ and effective ${ }^{240} \mathrm{pu}$ masses of nearly $600 \mathrm{~g}$ are shown in Table $\mathrm{I}$. The effective ${ }^{240} \mathrm{Pu}$ masses were calculated from the formula 1,6 .

$$
240 p_{u_{e f f}}=2.49\left({ }^{238} P_{u}\right)+\left({ }^{240} P_{u}\right)+1.57\left({ }^{242} p u\right) .
$$

Further descriptions of these sample compositions and containers are given in Ref. 6 . The declared mass density for these samples is $2.5 \mathrm{~g} / \mathrm{cm}^{3}$. However, because the largest sample (Puo, mass $=3 \mathrm{~kg}$ ) had to be tapped lightly to make it fit in the container, a mass density of $1.8 \mathrm{~g} / \mathrm{cm}^{3}$ is probably more realistic. Coincidence count rates for these samples are shown by the solid curve. in Fig. 1 .

Because a discrepancy exists in the sample mass density, correction factors were calcuiated from MCNP using both the 1.8 and $2.5 \mathrm{~g} / \mathrm{cm}^{3}$ densities. Results of the calculations are shown in Fig. 2, along with corresponding experimentally determined correction factors. The experimental values are taken from Ref. 6. 
TABLE I

$\mathrm{PUO}_{2}$ STANDARDS DATA

$\begin{array}{ccc}\text { 0xide Mass }(g) & \text { Pu Mass }(g) & 240 \text { pu effective }(g) \\ 500 & 436.2 & 97.45 \\ 1000 & 872.7 & 195.0 \\ 1500 & 1308.5 & 292.3 \\ 2000 & 1744.8 & 389.8 \\ 2500 & 2181.7 & 487.4 \\ 3000 & 2615.3 & 584.3\end{array}$

PU ISOTOPIC COMPOSITION

I sotope

238

239

240

241

242

wt\% on 2 0ct. 1979

0.140

75.340

20.947

2.908

0.666

241 Am content: $2530 \mathrm{ppm}$ in January 1977. Tap density: $2.5 \mathrm{~g} / \mathrm{cm}^{3}$.

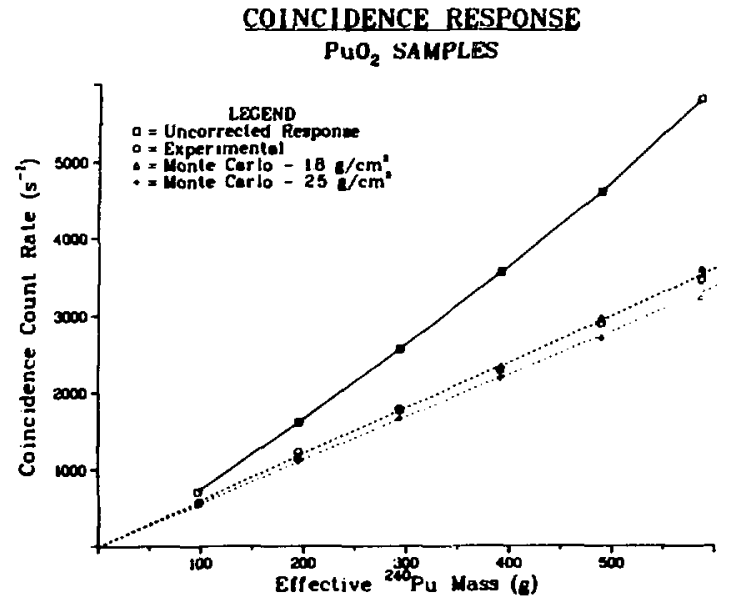

Fig. 1 . Coincidence rate vs $240 \mathrm{Pu}-$ powder samples. The solid curve represents the uncorrected count rate. The dashed curves represent corrected count rates obtained from three correctionfactor sets. (Note: Two of the corrected curves have identical slopes.)

\section{COINCIDENCE CORRECTION FACTORS} $\mathrm{PuO}_{2}$ SAMPLES

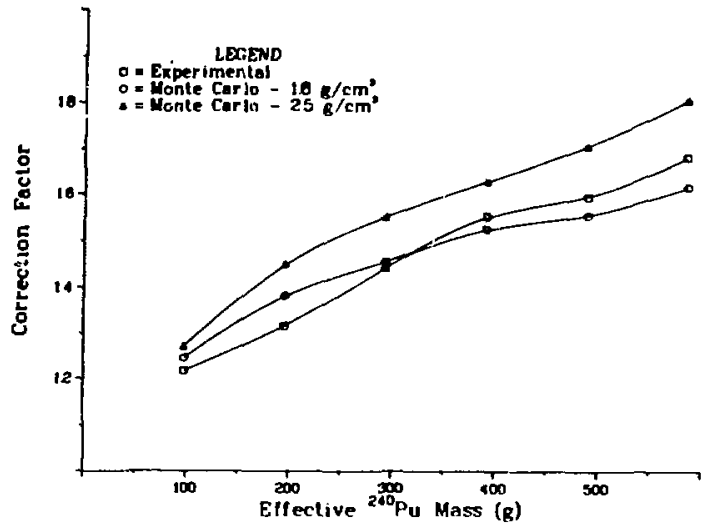

Fig. 2 .

Correction-factor sets for large mass $\mathrm{PuO}_{2}$ powder samples obtained from experimental means and from calculational methods using two different mass densities. 
When data shown in Fig. 2 are used to correct experimental count rates for sample multiplication, that is,

$$
\frac{\dot{R}}{C_{f}}=\dot{R}_{c},
$$

where $\dot{R}$ is the uncorrected count rate and $\dot{R}_{c}$ is the corrected count rate, resulting linear responses are shown by the dotted lines in Fig. 1. For these $\mathrm{PuO}_{2}$ samples, all three correctionfactor data yield linear corrected curves. The $1.8-\mathrm{g} / \mathrm{cm}^{3}$ corrested count rate set has the same slope as data corrected using experimental correction-factor techniques.

Ideally, the coincidence count rate should be directly propertonal to the material mass (M), that is,

$$
\dot{R}=\dot{R}_{0} M,
$$

where $\dot{R}_{0}$ is the count rate per unit mass. In reality, because of sample multiplication and the SR electronics used in the HLNCC and AwCC detectors, the true response is

$$
\dot{R}=\dot{R}_{0} M C_{f}(M) \text {, }
$$

where $C_{f}(M)$ is the mass-dependent correction factor. Several variations of $\mathrm{Eq}$. (3) can be used in understanding coincidence responses, that is,

$$
\begin{aligned}
& \dot{R}_{0}=\dot{R} / M C_{f}(M)=\dot{R}_{C} / M, \\
& M=\dot{R} / \dot{R}_{0} C_{f}(M)=\dot{R}_{C} / \dot{R}_{0}, \text { and } \\
& \dot{R} / \dot{R}_{0}=M C_{f}(M) .
\end{aligned}
$$

8 
In Eq. (4), correction-factor data are used to determine the normalized absolute detector courit rate. In Eq. (5), the mass of the material can be determined if $\dot{R}_{0}$ for a particular correction factor curve is known. Equation $(6)$ is used to separate basicaliy experimental data $\left(\dot{R} / \dot{R}_{0}\right)$ from calculational data $\left[M C_{f}(M)\right]$. For the calculationally assisted calibration, Eq. (6) is used. The quantity $M C_{F}(M)$ is calculated while $\dot{R}$ and $\dot{R}_{0}$ are measured.

For the large $\mathrm{PuO}_{2}$ samples, $\dot{R}_{0}$ values calculated from Eq. (4) for the two calculated and one experimental correction-factor sets are shown in Table II. Identical average $\dot{R}_{0}$ values were obtained for the experimental and $1.8-\mathrm{g} / \mathrm{cm}^{3}$ correction-factor sets. The average $\dot{R}_{0}$ value for the $2.5-\mathrm{g} / \mathrm{cm}^{3}$ set was 6.5 percent lower. In Fig. 3 , the individual $\dot{R}_{0}$ values for each correction-factor set are shown with the respective average values. All values are within \pm 5 percent of the average value and in most cases the numbers are within 2 percent.

Results obtained from using average values in Eq. (5) to calculate effective ${ }^{240} \mathrm{Pu}$ masses are shown in Table III. Also shown in Table III are masses obtained by averaging the $1.8-\mathrm{g} / \mathrm{cm}^{3}$ and $2.5-\mathrm{g} / \mathrm{cm}^{3}$ masses. As is seen from the data in Table III, all three correction-factor sets yield comparable results that are quite good. The average values obtained from the two calculated correction-factor sets are in excellent agreement with the known masses.

These results, as well as those ciescribed in Ref. 5, demonstrate the validity of Monte Carlo techniques for predicting coincidence counter multiplication for $\mathrm{PuO}_{2}$ and similar materials. Large $\mathrm{PuO}_{2}$

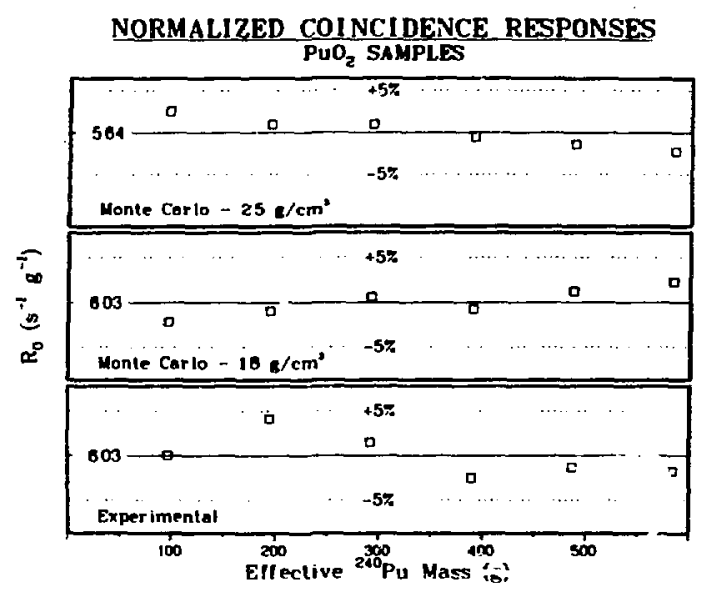

Fig. 3 .

Normalized reponses for large $\mathrm{PuO}_{2}$ powder samples using the three correction-factor sets. Dashed 1 ines show \pm 5 percent variance from the average values shown. 
TABLE II

$\dot{R}_{0}$ VALUES FOR VARIOUS CORRECTION-FACTOR SETS

\begin{tabular}{|c|c|c|c|c|c|c|c|c|}
\hline $\mathrm{PuO}_{2}$ & Effective & $\begin{array}{l}\text { Uncorrected } \\
\text { Count Rate }\end{array}$ & rorre & ection $F a c$ & ors & & $\dot{R}$ & \\
\hline $\begin{array}{l}\text { Mass } \\
\text { (g) }\end{array}$ & $\begin{array}{l}2{ }^{20} \mathrm{pu}_{\mathrm{pu} \text { Mass }} \\
\text { (g) }\end{array}$ & $\begin{array}{c}\text { Count Rate } \\
\left(s^{-1}\right) \\
\end{array}$ & Experimental $^{6}$ & $1.8 \mathrm{~g} / \mathrm{Cm}^{3}$ & $2.5 \mathrm{~g} / \mathrm{cm}^{3}$ & Experimental & $1 . \mathrm{g}^{\circ} \mathrm{g} / \mathrm{cm}^{3}$ & $2.5 \mathrm{~g} / \mathrm{cm}^{3}$ \\
\hline 500 & 97.45 & 715.4 & 1.217 & 1.245 & 1.270 & 6.03 & 5.90 & 5.78 \\
\hline 1000 & 194.90 & 1609.4 & 1.315 & 1.382 & 1.450 & 6.28 & 5.97 & 5.69 \\
\hline 1500 & 292.35 & 2577.0 & 1.441 & 1.454 & 1.549 & 6.12 & 6.07 & 5.69 \\
\hline 2000 & 389.80 & 3553.6 & 1.550 & $i .523$ & 1.625 & 5.88 & 5.98 & 5.61 \\
\hline 2500 & 487.25 & 4617.4 & 1.592 & 1.553 & 1.703 & 5.95 & 6.10 & 5.56 \\
\hline 3000 & 584.70 & 5811.8 & 1.679 & 1.614 & 1.804 & 5.92 & 6.16 & 5.51 \\
\hline
\end{tabular}

and other similar samples that are routinely inspected by IAEA personnel can be calculationally modeled. The modeling does not have to be exact, as demonstrated by the good results in 11 III for both the $1.8-$ and $2.5-\mathrm{g} / \mathrm{cm}^{3}$ curves. Applications of calculational techniques for evaluating unknown samples are described in the next section.

\section{v. CALCULATIONALLY ASSIETED EVALUATION TECHNIQUES DESCRIPTION}

In general, HLNCC or AWCC evaluation techniques are most accurate when appropriate count rate vs mass calibration curves are

TABLE III

EFfECtive $2400_{u}$ masses Calculateo from average $\dot{R}_{0}$ Values

\begin{tabular}{|c|c|c|c|c|c|c|c|c|}
\hline \multirow{2}{*}{$\begin{array}{c}\text { Known } \\
240_{\mathrm{Pu}} \text { Mass } \\
(\mathrm{g}) \\
\end{array}$} & \multicolumn{2}{|c|}{ Experimental } & \multicolumn{2}{|c|}{$1.8 \mathrm{~g} / \mathrm{cm}^{3}$} & \multicolumn{2}{|c|}{$2.5 \mathrm{~g} / \mathrm{cm}^{3}$} & \multicolumn{2}{|c|}{ Average } \\
\hline & $\begin{array}{l}\text { Mass } \\
(\mathrm{g}) \\
\end{array}$ & Error & $\begin{array}{l}\text { Mass } \\
(g)\end{array}$ & * Error & $\begin{array}{r}\text { Mass } \\
(g) \\
\end{array}$ & Error & $\begin{array}{l}\text { Mass } \\
(g) \\
\end{array}$ & Error \\
\hline .97 .45 & 97.49 & 0.04 & 95.33 & -2.18 & 99.89 & 2.50 & 96.61 & 0.16 \\
\hline$: 95.00$ & 202.97 & 4.08 & 193.19 & -0.93 & 196.83 & 0.94 & 195.01 & 0.005 \\
\hline 292.30 & 296.57 & 1.46 & 294.00 & 0.58 & 295.07 & 0.95 & 294.54 & 0.77 \\
\hline 389.80 & 380.21 & -2.46 & 386.85 & -0.76 & 387.64 & -0.55 & 387.25 & -0.66 \\
\hline 487.40 & 480.99 & -1.31 & 493.16 & 1.18 & 480.63 & -1.39 & 486.90 & -0.10 \\
\hline 584.30 & 574.04 & -1.76 & 597.23 & 2.21 & 571.27 & -2.23 & 584.25 & -0.01 \\
\hline Average & EError & 0.01 & & 0.02 & & 0.04 & & 0.03 \\
\hline Average & |xError| & 1.85 & & 1.31 & & 1.43 & & 0.28 \\
\hline
\end{tabular}

- XError $=100 *\left(\frac{\text { Evaluated mass - Known mass }}{\text { Known mass }}\right)$ 
available. Often, however, for some material types, calibration curves either do not exist or are inappropriate. Curves are inappropriate for a variety of reasons, but principally because material parameters of the sample being evaluated differ from corresponding parameters of the standard samples.

For evaluatior situations where calibration data are unavailable, calculationally generated data can be used. All that is required is 1) a normalization sample and 2) a set of correctionfactor calibration curves such as shown in Figs. 4 and 5 . These curves are extensions of the $\mathrm{PuO}_{2}$ data described in Sec. IV. The curves in $\mathrm{Fig}$. 4 represent third-order polynomial fits, that is,

$$
c_{f}=1.0+a_{1} M+a_{2} M^{2}+a_{3} M^{2},
$$

to the calculated data points where $C_{f}$ is the correction factor

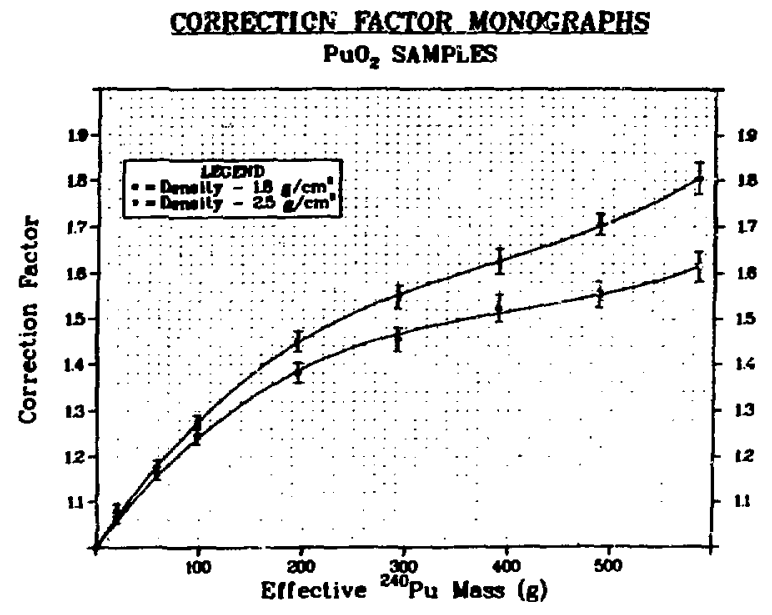

Fig. 4 .

Monograph of correction-factor data for large $\mathrm{PuO}_{2}$ samples. For these curves, two sample mass densities were considered.

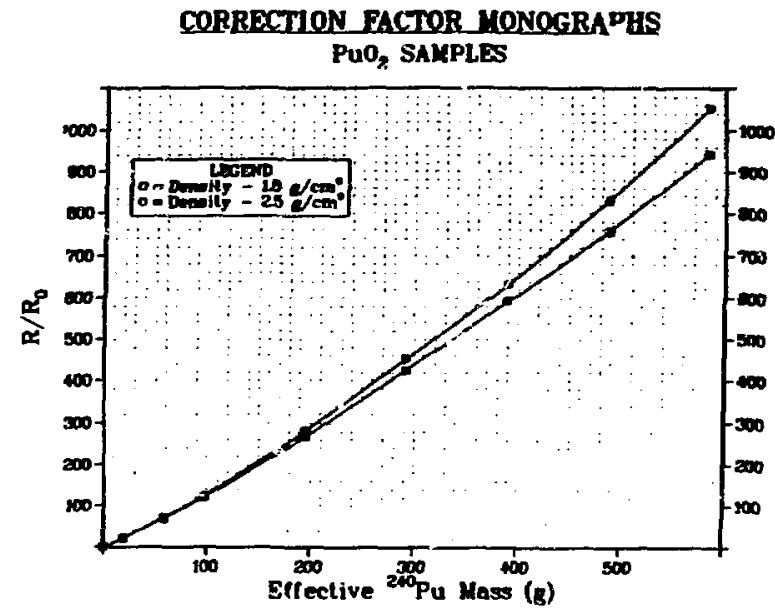

Fig. 5 .

falibration curves relating R $240 \mathrm{Pu}_{u}$ mass of a samp?e. $\dot{R}$ is the uncorrected count rate obtained from the counter. is the normalized coincidence response obtained for a given sample type and a given set of correction-factor data. 
and $M$ is the effective $240_{p u}$ mass. For the $1.8-\mathrm{g} / \mathrm{cm}^{3}$ curve, the coefficients are

$$
\begin{aligned}
& a_{1}=3.03 \times 10^{-3}, \\
& a_{2}=-6.46 \times 10^{-6}, \text { and } \\
& a_{3}=5.26 \times 10^{-9} .
\end{aligned}
$$

Corresponding values for the $2.5-\mathrm{g} / \mathrm{cm}^{3}$ curves are

$$
\begin{aligned}
& a_{1}=3.44 \times 10^{-3}, \\
& a_{2}=-7.05 \times 10^{-6}, \text { and } \\
& a_{3}=6.04 \times 10^{-9} .
\end{aligned}
$$

Error bars in Fig. 4 represent one standard deviation in the ca?culated values. In Fig. 5, $\dot{R} / \dot{R}_{0}$ yalues described in Eq. (6) are plotted for the two correction-factor sets.

With calibration curves such as those shown in Fig. 5, masses for unknown samples are found directly from the ratio of the measured count rate $(\dot{R})$ to the normalized count rate ( $\left.\dot{R}_{0}\right)$ once $\dot{R}_{0}$ is determined. Because $\dot{R}_{0}$ depends oil the specific detector being used and to a lesser degree on the type of material being measured, techniques are required for establis!ing $\dot{R}_{0}$. The simplest way to obtain $\dot{R}_{0}$ for a detector is to measure a very small sample that has little or no multiplication. For such a sample, the correction factor $\left(C_{f}\right)$ is approximately one; and from Eq. (4), $\dot{R}_{0}$ is then equal to the meacured count rate divided by the effective mass of the sample. In principle, this $\dot{R}_{0}$ value inherent to the detector and a-justed for a particular series of measurements with the ${ }^{252} \mathrm{Cf}$ normalization is adequate for all samples. Certainly in the absence of any other normalization, this value can and should be used. The main problem associated with using this inherent value of $\dot{R}_{0}$ is in applying correction-factor curves that approximate actual sample parameters. If the parameters used in selecting correction factor data are well defined and a curve that basically satisfies these parameters is avaisable, then the inherent detector $\dot{R}_{0}$ should yield very accurate mass 
results from fig. 5 applications. If, however, parameters used to generate a correction-factor curve do not match assay material parameters we11, significant errors can occur from using the inherent $\dot{R}_{0}$. To compensate for situations where sample parameters are not well-characterized by a correction-factor curve, a detectormaterial-dependent value for $\dot{R}_{0}$ can be determined. This normalization technique is contingent on the availability of a known sample of the material being measured or of a very similar material. With this known sample, an appropriate correction-factor curve is selected from Fig. 4-type data. A correction factor for the known sample is found from $F i g .4$ and $\dot{R}_{0}$ is determined from Eq. (4). From this $\dot{R}_{0}$ value and the data in Fig. 5, the unknown samples can be assayed by determining $\dot{R} / \dot{R}_{0}$ and then finding the appropriate mass. The accuracy of the detector-material normalization depends on (a) how well $\dot{R}_{0}$ is established for a given set of correction-factor data and (b) how well ine material being measured is characterized. One method for reducing errors is to obtain average masses from two material-detector normalizations using correction-factor data that bracket estimated material parameters.

As an example of how the technique is used, corisider the large $\mathrm{PuO}_{2}$ samples described in Table I. Assume that the sample with an effective ${ }^{240} \mathrm{pu}$ mass of $292.3 \mathrm{~g}$ is the known standard to be used for normalization. From Table Il, the coincidence count rate for this sample is $2577.0 \mathrm{~s}^{-1}$. From Fig. 4 it is seen that, for a density of $1.8 \mathrm{~g} / \mathrm{cm}^{3}$ and an effective $240 \mathrm{Pu}$ mass of $292.3 \mathrm{~g}$, the correction factor is 1.465. Similarly, for the $2.5-\mathrm{g} / \mathrm{cm}^{3}$ curve in Fig. 4, a 292.3-g mass has a correction factor of 1.552 . From Eq. (4), that is,

$$
\dot{R}_{0}=\dot{R} / M C_{f}(M) \text {, }
$$

the $1.8-\mathrm{g} / \mathrm{cm}^{3}$ and $2.5-\mathrm{g} / \mathrm{cm}^{3}$ density correction factors for the $292.3-g$ mass yield $\dot{R}_{0}$ values of $6.02\left(\mathrm{~s}^{-1} \mathrm{~g}^{-1}\right)$ and $5.68\left(\mathrm{~s}^{-1} \mathrm{~g}^{-1}\right)$, respectively. With these $\dot{R}_{0}$ values, $\dot{R} / \dot{R}_{0}$ quantities for all the samples in Table I are calculated as shown in Table IV. These 
$\dot{R} / \dot{R}_{0}$ vaiues are then used along with the curves in $F i g .5$ to determine the sample masses. The $\dot{R} / \dot{R}_{0}$ values obtained from the $1.8-\mathrm{g} / \mathrm{cm}^{3}$ correction-factor set, that is, $\dot{R}_{0}=0.02 \mathrm{~s}^{-1} \mathrm{~g}^{-1}$, are used on the upper curve of $F i g .5$ to determined masses. Similarly, the $2.5-\mathrm{g} / \mathrm{cm}^{3} \dot{R} / \dot{R}_{0}$ quantities are applicable to the lower curve on Fig. 5. Masses obtained from Fig. 5 are shown in Table IV. Also shown in Table IV are averayes of the masses obtained from the $1.8-\mathrm{g} / \mathrm{cm}^{3}$ and $2.5-\mathrm{g} / \mathrm{cm}^{3}$ correction-factor data.

As is seen, all three sets of mass values shown in Table IV are in excellent agreement with the known masses. The samples analyzed in this example were very well characterized in composition, in container geometry, and in detector configuration. From this information, accurate correction factors were calculated resulting in the excellent agreement between predicted and actual mass.

Two sets of correction factors, that is, for $1.8-9 / \mathrm{cm}^{3}$ and $2.5-\mathrm{g} / \mathrm{cm}^{3}$ mass densities, were used in this example to illustrate that excellent results can be obtained even if all parameters are not known exactly. The reason either set yiflds such good results is because both sets were applicable over the mass range of interest and because of the normalizations. Normalization of each correction factor set to experimenta? values compensates for di $i$ ferences between actual sample parameters and parameters used in

TABLE IV

EYAI.UATED MASSES IISIMG DETECIOR-MATERIAL NORMALIZATION

\begin{tabular}{|c|c|c|c|c|c|c|c|c|}
\hline \multirow{2}{*}{ 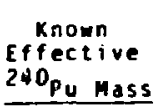 } & \multicolumn{2}{|c|}{$\dot{R}_{i} \dot{R}_{0}$} & \multicolumn{5}{|c|}{ Mass } & \multirow[b]{2}{*}{ Eerror } \\
\hline & $1.8 \mathrm{~g} / \mathrm{cm}^{3}$ & $2.5 \mathrm{~g} / \mathrm{cm}^{3}$ & $1.8 \mathrm{~g} / \mathrm{cm}^{3}$ & Error & $2.5 \mathrm{~g} / \mathrm{Cm}^{3}$ & Error & Averoge & \\
\hline 97.45 & 118.84 & 125.95 & $95 . \mathrm{C}$ & $\therefore 51$ & 98.0 & 0.36 & 96.5 & 0.97 \\
\hline 194.90 & 267.34 & 283.35 & 197.0 & $i .08$ & 195.0 & 0.05 & 196.0 & 0.56 \\
\hline 292.35 & 428.07 & 453.70 & 294.0 & 0.56 & 293.0 & 0.22 & 293.5 & 0.39 \\
\hline 369.80 & 590.30 & 625.63 & 390.0 & 0.03 & 387.0 & -0.72 & 388.5 & 0.33 \\
\hline 187.25 & 767.01 & 812.92 & 490.0 & 0.56 & 482.0 & -1.08 & 486.0 & -0.20 \\
\hline \multirow[t]{3}{*}{584.70} & 965,42 & 1023.20 & 596.0 & 1.93 & 574.0 & -1.83 & 385.0 & 0.05 \\
\hline & Aver: & EError & & 0.28 & & -0.47 & & 0.34 \\
\hline & Aver: & ge IIError & & 1.11 & & 0.34 & & 0.43 \\
\hline
\end{tabular}

aError - 100*

(Evaluated asss-Known mass) 
calculating correction-factor curres. These results demonstrate that a unique correction-factor curve is not needed for each sample type. However, accuracy of results obtained does depend on the normalization.

VI. GENERAL APPLICATIONS

A. Preparation of Appropriate Correction-Factor Monographs

For general applications of calculational correction-factor techniques, monographs of curves such as those shown in Figs. 6 and 7 could be prepared at Los Alamos for distribution to IAEA inspectors. Because of the total amount of computer time required to generate a set of correction-factor curves, parameters should not be varied arbitrarily. Instead, a narrow scope of values typical of the samples being evaluated by IAEA inspectors should be specified and then appropriate curves generated.

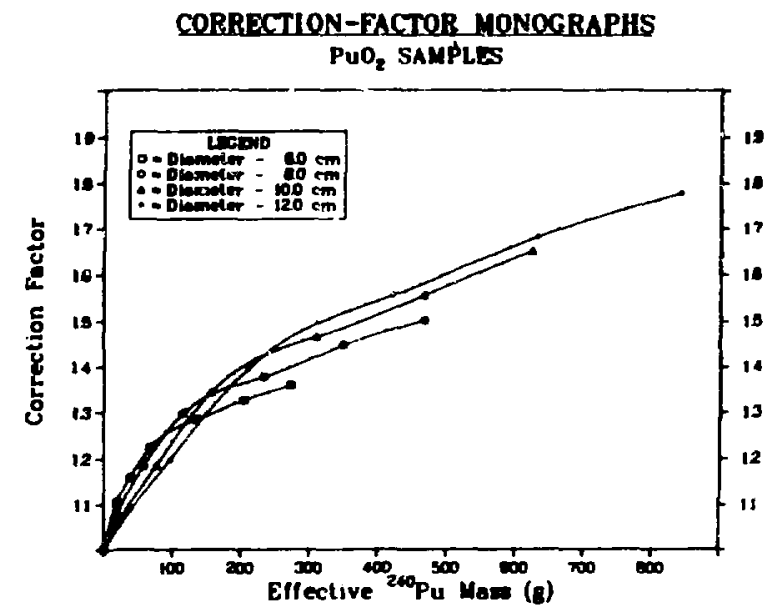

Fig. 6.

Generalized correction - factor data for large $\mathrm{PuO}_{2}$ powder samples. In these curves all parameters are fixed except the diameter of the sample. The diameter is varied over a range of possible container sizes.

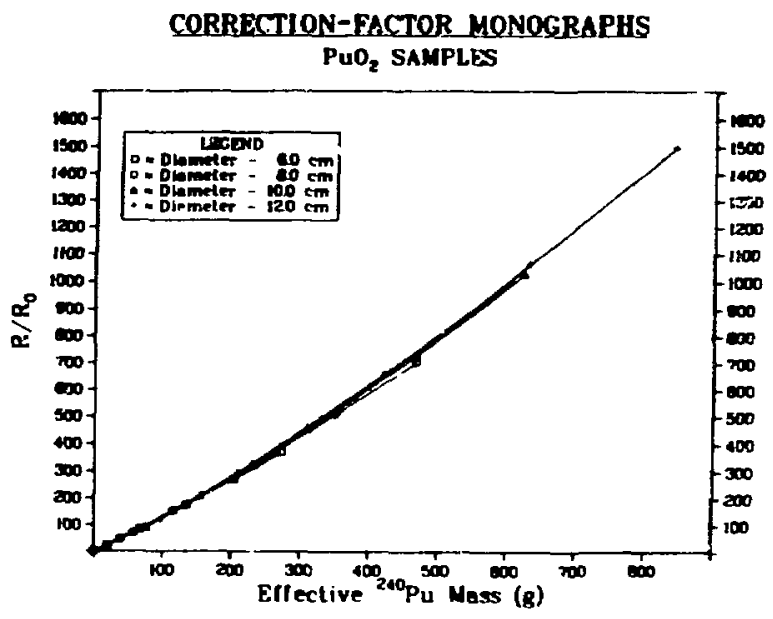

Fig. 7 .

Calibration curves corresponding to the correction-factor data in $\mathrm{Fig.} 6$. 
Three basic quantities, namely, mass density, geometry, and material composition, are needed for correction-factor monographs. Each monograph is generated by holding two of the parameters constant while varying the third. For example, in Figs. 6 and 7 , the geometry is varied for a fixed density of $1.8 \mathrm{~g} / \mathrm{cm}^{3}$ and a material composition shown in Table $I$.

Ranges of values for two of the quantities, that is, mass density and geometr.y, can be easily established from knowledge of the types of materials an IAEA inspector might want to measure clld of the types of containers in which these materials might be scored. For instance, for $\mathrm{UO}_{2}$ powders, a mass range of $1.5-3.0 \mathrm{~g} / \mathrm{cm}^{3}$ would probably be appropriate. Generally, these types of samples are contained in cylinders of varying radij ranging from $5-8 \mathrm{~cm}$. Thus, from these established ranges and from knowledge of material compositions, a set of monographs such as those in Figs. 6 and 7 could be generated that would be applicable to most vo 2 powder evaluations in an HLNCC. Similar types of curves could be prepared for other material types.

Specification of material composition parameters is more difficult than specifying mass density or geometry parameters. Knowledge of material composition is needed for specifying two sets of parameters in the Monte Carlo correction-factor calculations. First, the actual material specification used for the sample being modeled is determined from relative isotopic abundances. Multiplication and self-shielding effects in the sample are very dependent on these material composition specifications. Second, proportionate spontaneous fission and $(\alpha, n)$ neutron emission rates in the sample are also dependent on the isotopic composition. Correction fuctors for materials containing both spontaneous fission and $(\alpha, n)$ sources are very dependent on the relative emission rates from these sources. Thus; an accurate knowledge of the isotopic composition is needed and such compositions should be varied in a realistic marner. For instance, attempts to study $(a, n)$ emission rates without varying the isotopic composition of the material specirication would result in meaningless correction factors. Similar meaningless results would be obtained by varying the material 
specifications without also changing the ratio of spontaneous fission events to $(a, n)$ events. To vary these parameters in concert for arbitrary materials, some knowledge of the way isotopic compositions might change is needed. Such knowledge could be obtained from information shown in Fig. 8. In this figure, piutonium isotopic dependence on exposure is shown for typical pressurized water reactor (PWR) fuel. These data were calculated by the EPRI-CINDER codell for fuel from the $H$. B. Robinson unit 2 reactor. In the correction-factor calculations, such data, which represent actual relative isotopic compositions, should be used for varying $(a, n)$ and spontaneous fission neutron production rates and the corresponding material specifications. In this manner, the correlation between neutron emission rates and material composition can be accurately modeled as a function of exposure. For the correctionfactor monographs, data would then be generated for a given exposure range.

B. Inspector Use

Monographs like those in Figs. 6 and 7 can be used by IAEA inspectors to assist in evaluating unknown samples. If the density, geometry, and compositions are known for the sample being measured, then the appropriate correction-factor curve is chosen and the procedures in Sec. $V$ are performed to determine the effective 240 pu mass. If parameters are unknown, then estimates should be made in order to select a curve. For samples without well-defined characteristics, better results are obtainable if two correction-factor curves
CALCULATIONALIY ESTIMATED QUANTITIES PWR SENSITIVITY SERIES -- CASE A-2

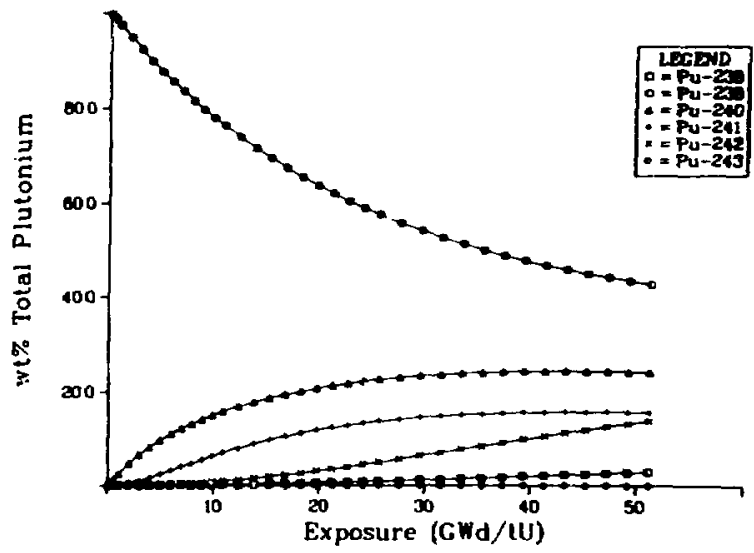

Fig. 8 .

Calculated buildup and depletion of the plutonium isotopes as a function of exposure. These data are for samples taken from the H. B. Robinson unit 2 reactor. 
with parameters that bracket estimated values are used. As shown in Sec. $V$, averaged masses obtained from applications of two or more correction-factor curves can yield more accurate results. In general, the overall acclicacy of the evaluation will depend on how well correction-factor curves represent material parameters and on the normalization.

With these techniques, an inspector can evaluate samples for which calibration curves do not exist. Also, an inspector can use the techniques as a means of verifying results obtained directly from a calibration curve. Large discrepancies between masses obtained from the correction-factor method and from a calibration curve could indicate sample characteristics that are different from characteristics of the calibration standards. In this case, an inspector should attempt to resolve the differences by using other measurement techniques or by attempting to better quantify sample parameters.

Monographs of correction factor data could be used by inspectors in the field to evaluate samples as they are being measured. In this manner, a real time accountability is achieved. As stated earlier, the accuracy of the technique depends on how well correction-factor data correspond to actual sample parameters and on the normalization. If both conditions are well satisfied, then 1-2 percent accuracies are obtainable. Poorly satisfied conditions may result in accuracies between, 5 and 10 percent. For the $7 a t t e r$ case, accuracy can be improved by using average values obtained from applying more than one correction factor set, that is, choosing sets which bracket probable sample parameters. This method also provides a means for verifying masses obtained from other methods and for adjusting data to account for slight variations in sample parameters. The ability to verify masses from other methods improves the sensitivity of the instrument. Correction-factor techniques also increase detectability limits because materials for which generic calibration curves do not exist can still be evaluated.

In principle, the technique can be used for any sample type that might be evaluated in an HLNCC. However, because of probiems 
in calculating correction factors for highly multiplying materials, the dynamic range of the technique may be limited to materials that have correction factors of 5 or less.

\section{CONCLUSIONS}

When appropriate experimental calibration methods are unavailable, calculational techniques can be used to support coincidence counter evaluations of unkriown materials. Correction-factor curves accounting for sample multiplication effects can be prepared using Monte Carlo computer techniques. Such curves are used to adjust experimental data and determine material masses. Effective use of the correction-factor technique requires a physical sample with known characteristics for normalizing the correction-factor data. In selecting an appropriate correction-factor curve for the sample being evaluated, some knowledge (either known or estimated) of miaterial composition and geometry is required. For evaluations with estimated parameters or for evaluations where known parameters are not exactiy specified by a specific correction curve, average vaiues for the mass should be obtained by using more than one curve. In such situations, selected correction-factor curves should represent as nearly as possible actual sample parameters.

Accuracy associated with using correction-factor techniques depends on two factors: namely, how well normalizing samples represent materials being evaluated and how well sample parameters are known or estimated. Generally, 2 percent or better numbers can be obtained with proper normalization and material specifications. Poorly characterized parameters probably will result in errors of 5 to 10 percent if a single value is determined and 2 to 6 percent if average values are used.

Correction-factors calculated with Monte Carlo techniques are fairly accurate in representing samples with low or moderate multiplications. Because of sampling problems in the Monte Carlo calculations, correction factors for materials with high multiplications are generally overpredicted. Monographs of correctionfactor curves $c$ an be generated for various material compositions, 
geometries, and mass densities. However, some guidance is necessary in choosing parameters typical of the materials that would be evaluated by IAEA inspectors. Generating many curves is time consuming both in setting up the problems for execution on the computer and in actual computer execution time. Thus only curves representing realistic data should be generated.

\section{ACKNOWLEDGEMENTS}

The author is most grateful to jim Stewart for providing his Monte Carlo correction-factor technique. This report is based primarily on this Monte Carlo technique. Also acknowledged is Bob Schrandt for adapting the correction-factor technique to the latest version of MCNP. Much advice and help in formulating a calculational calibration technique was received from Merlyn Krick, John Foley, and Howard Menlove.

\section{REFERENCES}

1. M. S. Krick and H. O. Menlove, "The High-Level Neutron Coincidence Counter (HLNCC): Users' Manual," Los Alamos Scientific Laboratory report LA-7779-M (ISPO-53) (June 1979).

2. H. 0. Menlove, "Description and Operation Manual for the Active Well Coincidence Counter," Los Alamos Scientific Laboratory report LA-7823-M (ISPD-66) (May 1979).

3. J. E. Swansen, P. R. Collinsworth, and M. S. Krick, "ShiftRegister Coincidence Electronics System for Therma? Neutron Counters," Los Alamos Scientific Laboratory report LA-8319-MS (April 1980).

4. M. S. Krick, "Calculation of Coincidence Counting Efficiency for Shift-Register and OSDOS Coincidence Circuits," in "Nuclear Safeguards Research and Development Program Status Report, May-August 1977," J. L. Sapir, Compiler, Los Alamos Scientific Laboratory report LA-7030-PR (March 1978), Pp. 14-16.

5. N. Ensslin, J. Stewart, and J. Sapir, "Self-Multiplication Correction Factors for Neutron Coincidence Counting," Nucl. Mat. Management 8,60 (1979). 
6. C. Beets, F. Franssen, R. Carchon, P. Vanderheyden, M. de Carolis, A. Keddar, J. Nelson, M. Krick, G. Bosler, and H. Menlove, "Optimization of NDA Measurements in Field Conditions for Safeguards Purposes," CEN/SCK Centre d'Etude de L'Energie Nucleaire (Mol, Belgium) report BLG 543 (February $1981)$.

7. M. S. Krick, "Neutron Multiplication Corrections for Passive Thermal Neutron Well Counters," Los Alamos Scientific Laboratory report LA-8460-MS (ISPO-89) (July 1980).

8. Owen Deutsch, "Coincidence Counting Applications of Monte Carlo," Los Alamos Scientific Laboratory, Memorandum to G. Bosler (March 13, 1980).

9. J. L. MacDonald, "A Monte Carlo Neutronic and Electronic Model for Thermal-Neutron Coincidence Counting," Trans. Am. Ñuci. Soc. 32,635 , (June 1979).

10. Los Alamos Group X-6, "MCNP - A General Monte Carlo Code for Neutron and Photon Transport," Los Alamos Scientific Laboratory report LA-7396-M, Revised (November 1979).

11. T. R. England, W. B. Wilson, and M. G. Stamatelatos, "Fission Product Data for Thermal Reactors Part 2: Users' Manual ior EPRI-CINDER Code and Data," LoS Alamos Scientific Laboratory report LA-6746-MS (EPRI NP-356) (December 1976). 